\title{
Review of: "The use of oxygen hoods in patients failing on conventional high-flow oxygen delivery systems, the effects on oxygenation, mechanical ventilation and mortality rates in hypoxic patients with COVID-19. A Prospective Controlled Cohort Study"
}

\author{
Islem OUANES
}

Potential competing interests: The author(s) declared that no potential competing interests exist.

Comment on the manuscript by Dayya $D$ et al, entitled "The use of oxygen hoods in patients failing on conventional high-flow oxygen delivery systems, the effects on oxygenation, mechanical ventilation and mortality rates in hypoxic patients with COVID-19. A Prospective Controlled Cohort Study", Respiratory Medicine 179 (2021) 106312.

The research question is very interesting, reflecting a real practical problem in ICUs, assessing possible oxygenation/ventilation alternative modality in severe hypoxemic Covid-19 patients, to prevent intubation and invasive mechanical ventilation (MV), known to be associated with high mortality reaching $80 \%$ in severe Covid-19 patients. In this field, physicians are daily confronted due to many challenges: time and emergency of the decision, the lack of various alternative tools and modalities effective in preventing or suppling intubation and MV and their associated complications.

Hyperbaric oxygenation and Hoods have been demonstrated as effective in many other settings, few studies assessed their contribution in Covid-19 hypoxemic patients.

In the present study, authors found an oxygenation improvement as measured by $\mathrm{SaO} 2$ increase by $8.8 \%$ $(p<0.001)$, they reported that hoods averted imminent or immediate intubation/MV in intervention group, they finally suggested that in hospital MV and mortality rates were reduced (without statistically significant differences) with the use of these proposed devices.

The manuscript is well written and easy to read, most of statistical analyses are appropriate. Nevertheless, I have some concerns and comments with many points:

- First, I think that the appropriate term to describe the study design is "a before-after cohort study" more than "a prospective controlled cohort study"; the availability of hoods on 4/3/2020 in the institution was here determinant.

- Objectives and outcomes in this study were multiple, and risk factors assessment with logistic regression analysis was not sufficient to adjust confounding factors, a propensity score analysis could be a better solution for this aim. 
- Included population as defined by authors was "hypoxemic Covid-19 patients failing on conventional high-flow oxygen delivery systems", failing here means that those patients should be intubated at that moment. We would therefore logically expect an intubation rate of $100 \%$ in the control group, i.e. before the availability of hoods, as a potential alternative modality to avoid intubation, the reported MV rate in control group was only $47.4 \%$.

- Exclusion criteria: I think that excluding patients with confinement anxiety post-oxygen hood placement with removal request, was not appropriate, because this could lead to intubation and MV (in patients failing on conventional high-flow oxygen delivery systems), and analysis should be made with intention to treat (ITT). On the other hand including patients with Do Not Intubate (DNI) or Do Not Resuscitate (DNR) decisions may interfere with results and interpretation.

- In table 2 reporting COVID-19 demographic characteristics and coexisting conditions among intervention cohort receiving oxygen hoods and control cohort receiving conventional high flow oxygen delivery, we notice many imbalances between the two groups, favoring somewhere the intervention group. Age, 68 vs 70.4; BMI 28.2 vs 29.7; chronic comorbidities and immunosuppression were more prevalent in control group, and management was convalescent plasma was more used in the intervention group $(p<0.001)$. These imbalances even when not statistically significant they could include by a "cumulative effect" bias in patient's outcome assessment.

- To conclude that hoods averted immediate intubation in $100 \%$ of patients in the intervention group (58/58), we need a clear definition of "immediate intubation" (hours, 24 hours...), and intubation delay should be also reported in the two groups, to better distinguish between early and late failures.

- Impact on MV rates and mortality was not statistically significant, therefore, it cannot be concluded that these two events were reduced by the intervention (baseline imbalances); the only consistent effect in this study was the improvement of oxygenation.

\section{Dr Islem Ouanes}

\section{Intensive Care Unit}

Clinique EI Yosr Internationale, Sousse, TUNISIA 